\title{
Raloxifene reduces skeletal fractures in an animal model of osteogenesis imperfecta
}

Alycia G. Berman ${ }^{1}$, Joseph M. Wallace ${ }^{1,2}$, Zachary R. Bart ${ }^{1}$, and Matthew R. Allen ${ }^{1,2,3}$

${ }^{1}$ Department of Biomedical Engineering, Indiana University Purdue University of Indianapolis, and ${ }^{2}$ Department of Orthopedic Surgery and ${ }^{3}$ Department of Anatomy and Cell Biology, Indiana University School of Medicine and Indianapolis, IN, United States.

Keywords: OI, bone mechanical properties, SERM, oim/oim

Send Correspondence to:

Matthew R. Allen, PhD

Dept. of Anatomy and Cell Biology, MS 5035

Indiana University School of Medicine

635 Barnhill Dr.

Indianapolis, IN 46202

Tel: $\quad 317-274-1283$

FAX: $\quad 317-278-2040$

Email: matallen@iupui.edu 


\section{ABSTRACT}

Osteogenesis imperfecta $(\mathrm{OI})$ is a genetic disease of Type I collagen and collagenassociated pathways that results in brittle bone behavior characterized by fracture and reduced mechanical properties. Based on previous work in our laboratory showing that raloxifene (RAL) can significantly improve bone mechanical properties through non-cellular mechanisms, we hypothesized that raloxifene would improve the mechanical properties of OI bone. In experiment 1, tibiae from female wild type (WT) and homozygous oim mice were subjected to in vitro soaking in RAL followed by mechanical tests. RAL soaking resulted in significantly higher post-yield displacement $(+75 \%$ in WT, $+472 \%$ in oim; $p<0.004)$, with no effect on ultimate load or stiffness, in both WT and oim animals. In experiment 2, eight-week old WT and oim male mice were treated for eight weeks with saline vehicle (VEH) or RAL. Endpoint measures included assessment of in vivo skeletal fractures, bone density/geometry and mechanical properties. In vivo skeletal fractures of the femora, assessed by micro CT imaging, were significantly lower in oim-RAL (20\%) compared to oim-VEH $(48 \%, p=0.047)$. RAL led to significantly higher DXA-based BMD $(p<0.01)$ and CT-based trabecular BV/TV in both WT and oim animals compared to those treated with VEH. Fracture toughness of the femora was lower in oim mice compared to WT and improved with RAL in both genotypes. These results suggest that raloxifene reduces the incidence of fracture in this mouse model of oim. Furthermore, they suggest that raloxifene's effects may be the result of both cellular (increased bone mass) and non-cellular (presumably changes in hydration) mechanisms, raising the possibility of using raloxifene, or related compounds, as a new approach for treating bone fragility associated with OI. 


\section{INTRODUCTION}

Osteogenesis Imperfecta $(\mathrm{OI})$, is a heritable disorder of connective tissues caused by mutations in Type I collagen or other genes in collagen associated pathways [1,2]. Over 1500 independent alterations in the primary structure of OI collagen have been identified leading to a wide spectrum of clinical severities. The dominant OI phenotype is in bone and is typified by low energy fracture, leading to the term "brittle bone" disease. Ol compromises the three main components that influence bones' mechanical properties - bone mass, geometry, and bone quality [3-8]. The complex changes across multiple-hierarchical levels present unique challenges for treatment.

Several mouse models of OI exist [9]. The Osteogenesis Imperfecta murine (oim) model was described in 1993 [10], and is the most characterized of these models. This mouse has a single base pair mutation in the $\alpha 2$ gene causing a frame shift of the final 48 amino acids at the C-terminus of the propeptide. This shift changes the collagen amino acid sequence and adds an extra residue. The resulting $\alpha 2$ chains are non-functional as they can no longer associate with $\alpha 1$ chains [1]. Homotrimeric $\alpha 1$ collagen molecules result, accumulating in the extracellular matrix and disrupting proper fibril assembly. $\alpha 2$ gene mutations are rare in humans, but the homozygous oim mouse is considered a good phenotypic model of human type III OI, as it results in a moderate to severe OI characterized by spontaneous fractures and limb deformities [11].

Current pharmacological interventions for OI incompletely normalize mechanical properties. For many forms of OI, pharmacological treatment with bisphosphonates has proven effective in reducing, but not eliminating fracture as assessed with cross sectional $[12,13]$ and controlled clinical trials [14-19]. When trials were examined with meta-analysis it was clear that a treatment gap remains as clear evidence of improved clinical status is lacking [20]. 
Animal data (using both the oim and Brtl/+ animal models) have documented that treatment with bisphosphonates can recover bone mass and architecture to near wild-type levels, yet whole-bone mechanical properties remain compromised compared to normal bone [21-23]. This is likely due to the lack of positive effect of bisphosphonates on the quality of the bone tissue [24]. More recent data with anabolic treatment (anti-sclerostin antibody) have also shown positive results in animal models (specifically the Brtl mouse), but again, the data point to drug-induced effects on bone mass, but not quality [25]. Thus, current approaches have been able to enhance bone mass in the setting of OI but have failed to positively affect bone quality.

Raloxifene, a selective estrogen receptor modulator (SERM), is an FDA-approved agent used to reduce fracture risk in osteoporotic patients [26,27]. SERMs are utilized as they effectively antagonize estrogen receptors in bone cells (reducing osteoclast development) while not affecting other estrogenic tissues. Recent work has shown that raloxifene, both in vivo and in vitro, increases bone through an additional mechanism, specifically through non-cellular effects that result in increased skeletal bound water [28,29]. The raloxifene-induced alterations in bound water are associated with positive effects on bone mechanical properties $[28,29]$ and these benefits

have recently been shown to extend to disease models with compromised bone quality [30]. Therefore, the goal of this study was to test the hypothesis that raloxifene could produce beneficial effects on bone mechanical properties in a mouse model of OI.

\section{RESULTS}

Experiment \#1

Paired tibiae from WT and oim mice were mechanically tested to failure in 4-point bending after ex vivo incubation in either PBS or RAL. Force (yield and ultimate), displacement (yield, post-yield and total), stiffness, and work (yield, post-yield, and total) were all significantly lower 
with oim (Table 1). RAL soaking had a significant main effect, producing higher post-yield displacement $(p<0.0001)$, and total displacement $(p<0.0001)$ (Table 1 and Figure 1). This indicates that RAL is able to impart benefits to mechanical properties through non-cellular mechanisms in both WT and oim mouse bones. For properties that had a significant interaction effect, significant differences $(p<0.001$ for all) in yield force, displacement to yield, stiffness, and work to yield were observed with RAL soaking in the WT group, but not in the oim group. Total work was significantly different with RAL treatment in both the WT and oim groups.

Figure 1

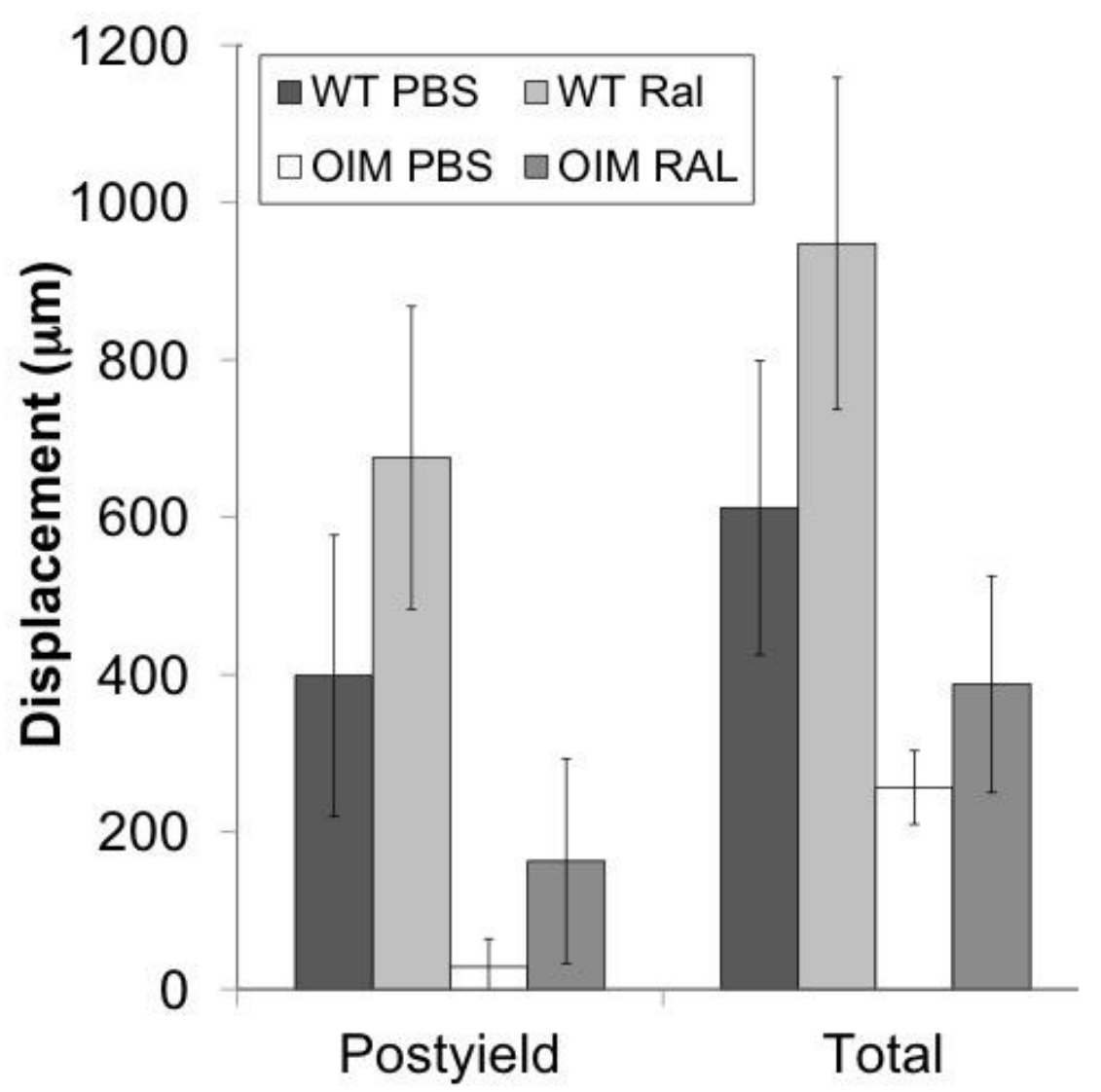

Figure 1. Ex-vivo soaking of osteogenesis imperfecta bones in raloxifene leads to significantly higher mechanical properties. In this $2 \times 2$ study design, both post-yield displacement and total displacement had significant main effects of disease and treatment (each $p<0.05)$ indicating that oim bones were lower than WT for both properties and that raloxifene exposure led to significantly higher properties in both wild-type and oim animals. Values are presented as 
mean \pm standard deviation. WT- wild-type; oim-osteogenesis imperfecta; PBS-phosphate buffered saline; RALraloxifene.

Table 1: Mechanical properties from 4-point bending of raloxifene-soaked tibiae

\begin{tabular}{|c|c|c|c|c|}
\hline & \multicolumn{2}{|c|}{ WT } & \multicolumn{2}{|c|}{ OIM } \\
\hline & Control $(n=15)$ & Raloxifene $(n=15)$ & Control $(n=9)$ & Raloxifene $(\mathrm{n}=9)$ \\
\hline Yield Force $(\mathrm{N}){ }^{\#}$ & $18.40 \pm 1.25^{a b}$ & $22.17 \pm 2.01^{\text {ad }}$ & $6.77 \pm 1.35^{b}$ & $6.96 \pm 1.64^{d}$ \\
\hline Ultimate Force $(\mathrm{N})^{{ }^{*}+}$ & $23.44 \pm 2.92$ & $24.65 \pm 1.79$ & $7.28 \pm 1.64$ & $8.00 \pm 1.65$ \\
\hline Stiffness $(\mathrm{N} / \mathrm{mm})^{\#}$ & $105.2 \pm 10.17^{a b}$ & $94.09 \pm 12.42^{\text {ad }}$ & $35.27 \pm 35.27^{b}$ & $36.37 \pm 7.27^{d}$ \\
\hline Deformation to Yield $(\mu \mathrm{m}){ }^{\#}$ & $213 \pm 16^{a}$ & $272 \pm 33^{\text {ad }}$ & $228 \pm 23$ & $224 \pm 18^{d}$ \\
\hline Postyield Deformation $(\mu \mathrm{m})^{{ }_{+}}$ & $398 \pm 178$ & $675 \pm 192$ & $28 \pm 35$ & $163 \pm 130$ \\
\hline Total Deformation $(\mu \mathrm{m})^{*+}$ & $611 \pm 186$ & $947 \pm 210$ & $256 \pm 46$ & $387 \pm 137$ \\
\hline Work to Yield $(\mathrm{mJ}){ }^{\#}$ & $2.06 \pm 0.20^{a b}$ & $3.19 \pm 0.60^{\text {ad }}$ & $0.83 \pm 0.21^{b}$ & $0.86 \pm 0.25^{d}$ \\
\hline Postyield Work (mJ) ${ }^{*+}$ & $8.12 \pm 3.05$ & $14.56 \pm 3.96$ & $0.21 \pm 0.26$ & $1.18 \pm 0.80$ \\
\hline Work to Failure $(\mathrm{mJ}){ }^{\#}$ & $10.18 \pm 3.16^{a b}$ & $17.75 \pm 4.22 \mathrm{ad}$ & $1.04 \pm 0.39 \mathrm{bc}$ & $2.04 \pm 0.86^{c d}$ \\
\hline
\end{tabular}

Values are presented as mean \pm standard deviation. WT- wild-type; oim-osteogenesis imperfecta. In the property column, * indicates a main effect of disease, ${ }^{+}$indicates a main effect of treatment, and \# indicates an interaction term ( $\left.p<0.05\right)$. For parameters with an interaction term, a posthoc pairwise t-test with Bonferroni correction was used ( $p<0.0125)$. ${ }^{a}$ indicates a significant difference between WT-Control and WT-Raloxifene, ${ }^{b}$ indicates a significant difference between WT-Control and oim-Control, ${ }^{c}$ indicates a significant difference between oim-Control and oim-Raloxifene, and d indicates a significant difference between WT-Raloxifene and oim-Raloxifene.

\section{Experiment \#2}

\section{Fracture Assessment}

There were no fractures in the WT animals. In oim vehicle-treated animals, $48 \%$ of the femurs were fractured, while in the oim raloxifene-treated animals the percentage of fractures was significantly lower (20\%, $\mathrm{p}=0.047)$ (Figure 2). 
Figure 2
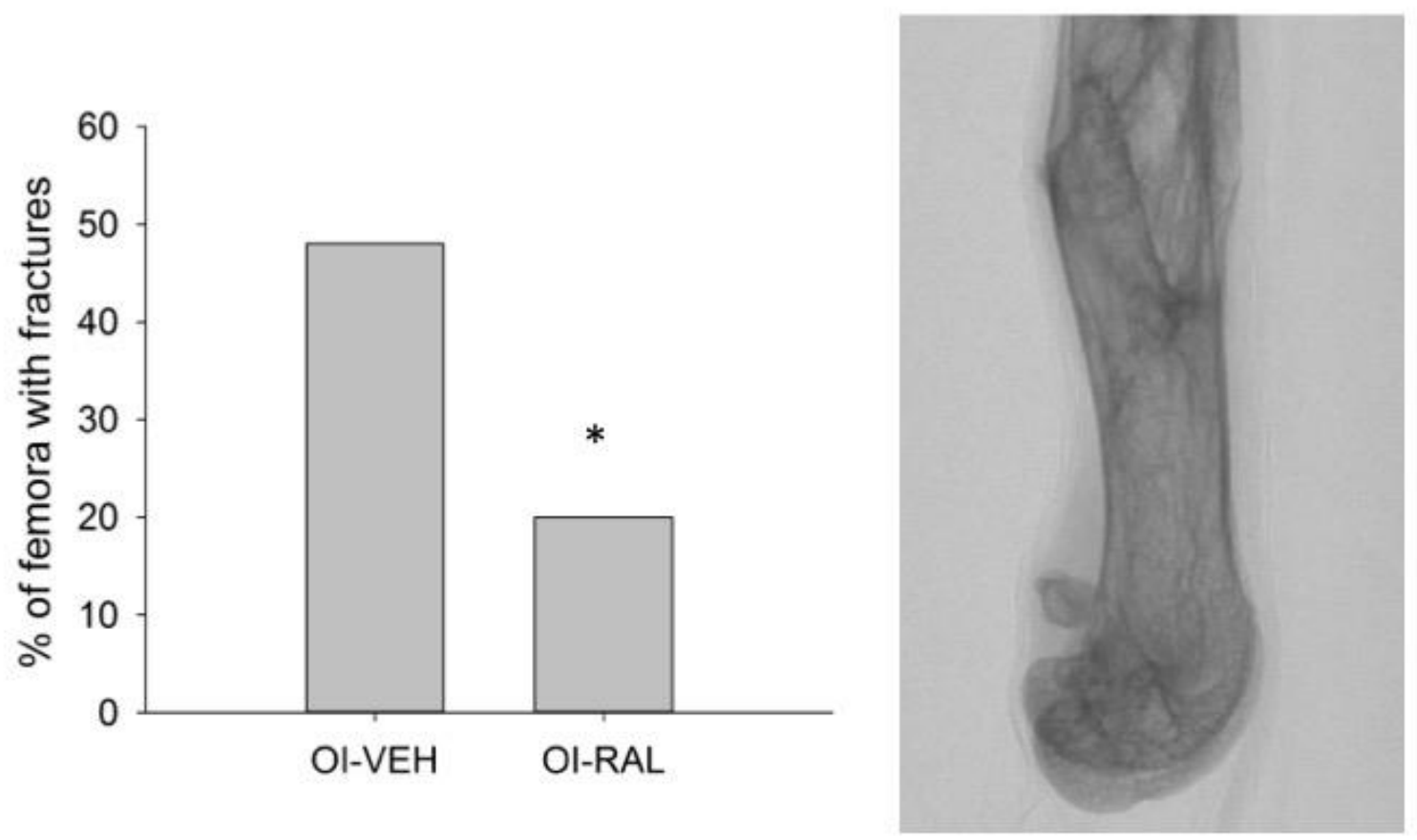

Figure 2. In vivo treatment with raloxifene lead to significantly fewer femoral fractures in animals with osteogenesis imperfecta. In vehicle-treated animals, $48 \%$ of the femora (13 of 27 ) were fractured (one bone was missing from the analysis). In raloxifene-treated animals, $20 \%$ of the femora (6 of 30$)$ were fractured. This represents a significant $\left({ }^{*} \mathrm{p}\right.$ $<0.05) 58 \%$ reduction in the number of in vivo fractures. A representative projection image of a fractured bone from an oim animals is depicted.

\section{$D X A$}

DXA-based BMD in the oim mice was significantly lower for the whole body, L4-L5 vertebrae, and femur compared to WT ( $p<0.001$ each). RAL treatment resulted in higher BMD in both genotypes as indicated by a significantly higher BMD in the whole body (WT: $+4 \%$, oim: $+7 \% ; p=0.0187)$ and femur (WT: $+10 \%$, oim: $+13 \% ; p=0.0066)$. Oim animals treated with RAL remained lower than WT controls for all sites (Figure 3). 
Figure 3

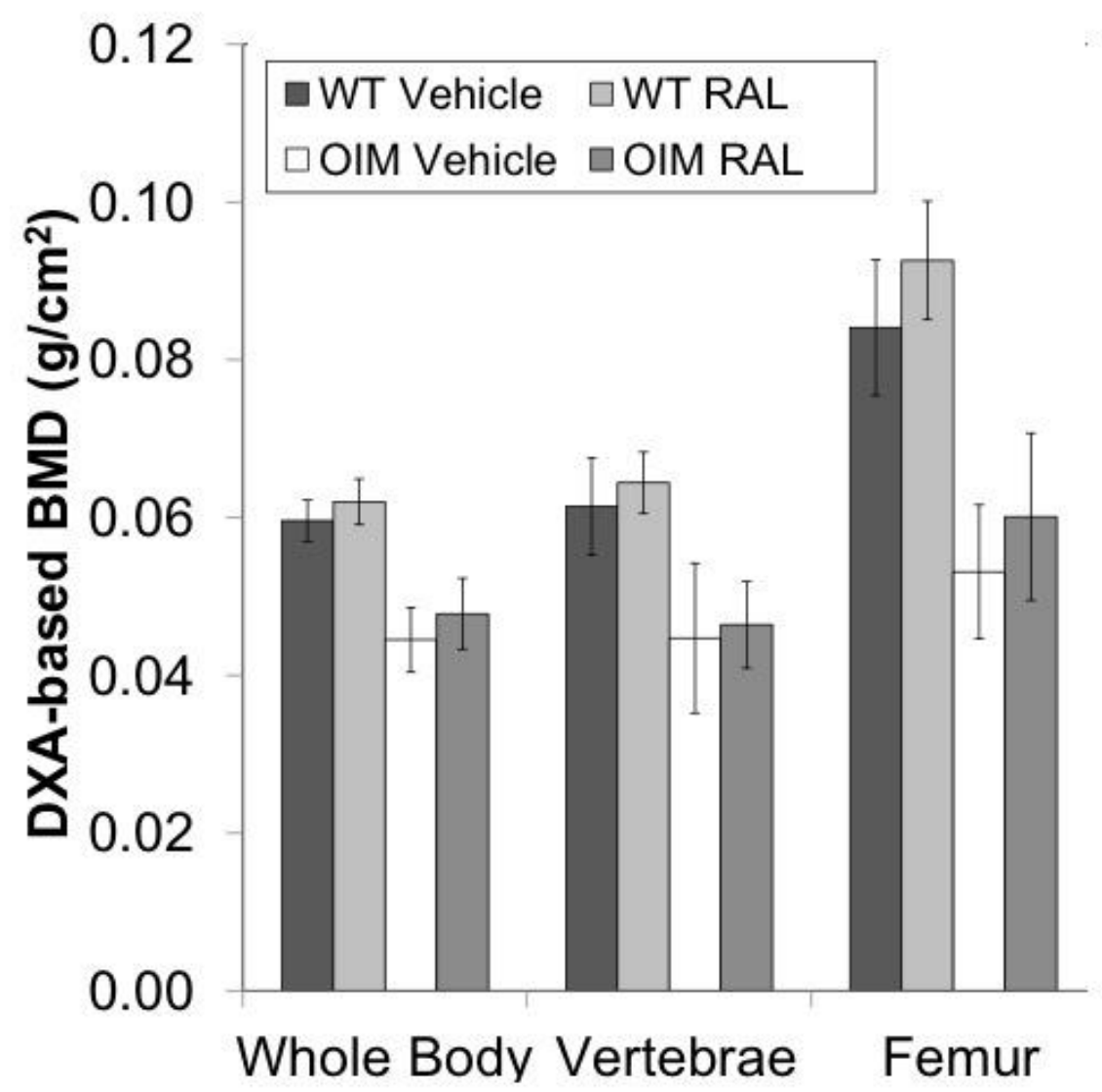

Figure 3. In vivo treatment with raloxifene resulted in higher areal bone mineral density in animals with osteogenesis imperfecta. Following 8 weeks of treatment, total body, lumber spine, and femoral bone mineral density (BMD) from dual-energy x-ray absorptiometry was assessed. In this $2 \times 2$ study design, all three measures had significant main effects of disease $(p<0.05)$, while whole body and femur also had main effects of treatment $(p<0.05)$, indicating that oim bones were lower than WT and that raloxifene exposure led to significantly higher properties in both wild-type and oim animals. Values are presented as mean \pm standard deviation. WT- wild-type; oim-osteogenesis imperfecta; RAL-raloxifene.

uCT

Within the cancellous ROI, bone volume per tissue volume (BV/TV), trabecular number (Tb.N), trabecular thickness (Tb.Th), trabecular spacing (Tb.Sp), and bone mineral density (BMD) were all significantly worse in the oim animals compared to WT ( $p<0.0001$ for all) (Table 2). With raloxifene treatment, BV/TV was significantly higher in both genotypes (WT: $+5 \%$, oim: $+68 \%$; 
$\mathrm{p}=0.0381$ ). These effects on BV/TV were mainly driven by differences in Tb. Th. In addition, BMD was significantly higher in RAL-treated animals (WT: $+2 \%$, oim: $+6 \% ; p<0.0001$ ).

Table 2: Cancellous architecture in the distal femoral metaphysis

\begin{tabular}{|l|c|c|c|c|}
\hline \multirow{2}{*}{} & \multicolumn{2}{|c|}{ WT } & \multicolumn{2}{c|}{ OIM } \\
\cline { 2 - 5 } & Control $(\mathrm{n}=8)$ & Raloxifene $(\mathrm{n}=8)$ & Control $(\mathrm{n}=10)$ & Raloxifene $(\mathrm{n}=12)$ \\
\hline BV/TV $(\%)^{*+}$ & $21.19 \pm 2.97$ & $22.16 \pm 3.97$ & $4.88 \pm 3.01$ & $8.20 \pm 3.00$ \\
\hline Trabecular thickness $(\mu \mathrm{m})^{*+}$ & $0.0669 \pm 0.0023$ & $0.0702 \pm 0.0028$ & $0.0544 \pm 0.0036$ & $0.0611 \pm 0.0031$ \\
\hline Trabecular separation $(\mathrm{mm})^{*}$ & $0.189 \pm 0.014$ & $0.186 \pm 0.013$ & $0.364 \pm 0.126$ & $0.300 \pm 0.068$ \\
\hline Trabecular number $(1 / \mathrm{mm})^{*}$ & $3.160 \pm 0.401$ & $3.143 \pm 0.459$ & $0.880 \pm 0.518$ & $1.331 \pm 0.459$ \\
\hline Tissue Mineral Density $\left(\mathrm{g} / \mathrm{cm}^{3}\right)^{*+}$ & $0.836 \pm 0.015$ & $0.855 \pm 0.019$ & $0.768 \pm 0.025$ & $0.814 \pm 0.027$ \\
\hline
\end{tabular}

Values are presented as mean \pm standard deviation. WT- wild-type; oim-osteogenesis imperfecta; BV/TV - bone volume/tissue volume. In the property column, ${ }^{*}$ indicates a main effect of disease and ${ }^{+}$indicates a main effect of treatment.

Analysis of a standard cortical ROI indicated that oim mice had significantly lower total cross sectional area $(p<0.001)$, cortical area $(p<0.001)$, cortical thickness $(p<0.001)$, periosteal and endocortical bone perimeters $(p<0.001$ and $p=0.002$, respectively), and moment of inertia in the maximum and minimum directions $(p<0.001$ for both) versus WT (Table 3 ). RAL treatment resulted in significantly higher cortical thickness in both genotypes $(p=0.0185)$.

Table 3: Cortical geometry at the femoral mid-diaphysis

\begin{tabular}{|l|c|c|c|c|}
\hline \multirow{2}{*}{} & \multicolumn{2}{|c|}{ WT } & \multicolumn{2}{c|}{ OIM Raloxifene $(n=12)$} \\
\cline { 2 - 5 } & Control $(\mathrm{n}=8)$ & Raloxifene $(\mathrm{n}=8)$ & Control $(\mathrm{n}=10)$ & $1.380 \pm 0.129$ \\
\hline Cross Sectional Area $\left(\mathrm{mm}^{2}\right)^{*}$ & $1.992 \pm 0.189$ & $1.985 \pm 0.225$ & $1.427 \pm 0.211$ & $0.726 \pm 0.111$ \\
\hline Marrow Area $\left(\mathrm{mm}^{2}\right)$ & $0.867 \pm 0.132$ & $0.794 \pm 0.101$ & $0.806 \pm 0.170$ & \\
\hline
\end{tabular}




\begin{tabular}{|l|c|c|c|c|}
\hline Cortical Area $\left(\mathrm{mm}^{2}\right)^{*}$ & $1.126 \pm 0.105$ & $1.191 \pm 0.133$ & $0.622 \pm 0.111$ & $0.654 \pm 0.090$ \\
\hline Cortical Thickness $(\mathrm{mm})^{*}$ & $0.273 \pm 0.022$ & $0.293 \pm 0.017$ & $0.171 \pm 0.026$ & $0.184 \pm 0.027$ \\
\hline Periosteal Perimeter $(\mathrm{mm})^{*}$ & $5.784 \pm 0.261$ & $5.768 \pm 0.290$ & $4.981 \pm 0.352$ & $4.967 \pm 0.272$ \\
\hline Endocortical Perimeter $(\mathrm{mm})^{*}$ & $4.274 \pm 0.330$ & $4.086 \pm 0.277$ & $3.863 \pm 0.371$ & $3.715 \pm 0.263$ \\
\hline $\operatorname{lmax}\left(\mathrm{mm}^{4}\right)^{*}$ & $0.375 \pm 0.078$ & $0.392 \pm 0.085$ & $0.146 \pm 0.050$ & $0.152 \pm 0.030$ \\
\hline $\operatorname{Im}\left(\mathrm{mm}^{4}\right)^{*}$ & $0.175 \pm 0.027$ & $0.179 \pm 0.040$ & $0.086 \pm 0.022$ & $0.081 \pm 0.020$ \\
\hline Tissue Mineral Density $\left(\mathrm{g} / \mathrm{cm}^{3}\right)$ & $1.271 \pm 0.016$ & $1.278 \pm 0.018$ & $1.281 \pm 0.032$ & $1.284 \pm 0.036$ \\
\hline
\end{tabular}

Values are presented as mean \pm standard deviation. WT- wild-type; oim-osteogenesis imperfecta; $I_{\max }$ Maximum moment of inertia; $I_{\min }$-Minimum moment of inertia. In the property column, * indicates a main effect of disease and + indicates a main effect of treatment.

\section{Whole Bone Mechanical Testing}

At both the structural and tissue level, oim bones exhibited significantly lower mechanical properties compared to WT ( $p<0.024$ for all properties) (Table 4). There were no significant effects of raloxifene treatment for any parameter.

Table 4: Tibial mechanical properties from 4-point bending

\begin{tabular}{|l|c|c|c|c|}
\hline \multirow{2}{*}{} & \multicolumn{2}{|c|}{ WT } & \multicolumn{2}{c|}{ OIM } \\
\cline { 2 - 5 } & Control $(\mathrm{n}=7)$ & Raloxifene $(\mathrm{n}=8)$ & Control $(\mathrm{n}=8)$ & Raloxifene $(\mathrm{n}=7)$ \\
\hline Yield Force $(\mathrm{N}){ }^{*}$ & $24.35 \pm 4.75$ & $27.66 \pm 3.99$ & $8.97 \pm 2.57$ & $8.79 \pm 3.02$ \\
\hline Ultimate Force $(\mathrm{N}){ }^{*}$ & $27.29 \pm 3.87$ & $28.62 \pm 4.48$ & $10.61 \pm 2.95$ & $10.87 \pm 3.53$ \\
\hline Displacement to Yield $(\mu \mathrm{m})^{*}$ & $184 \pm 28$ & $209 \pm 18$ & $175 \pm 23$ & $169 \pm 21$ \\
\hline Postyield Displacement $(\mu \mathrm{m})^{*}$ & $314 \pm 113$ & $263 \pm 160$ & $102 \pm 55$ & $97 \pm 18$ \\
\hline Total Displacement $(\mu \mathrm{m})^{*}$ & $498 \pm 132$ & $471 \pm 170$ & $277 \pm 69$ & $266 \pm 26$ \\
\hline Stiffness $(\mathrm{N} / \mathrm{mm})^{*}$ & $137.78 \pm 13.24$ & $141.26 \pm 14.93$ & $53.30 \pm 20.38$ & $55.24 \pm 22.96$ \\
\hline Work to Yield $(\mathrm{mJ}){ }^{*}$ & $2.38 \pm 0.82$ & $2.98 \pm 0.62$ & $0.80 \pm 0.23$ & $0.77 \pm 0.27$ \\
\hline Total Work $(\mathrm{mJ})^{*}$ & $8.81 \pm 2.96$ & $7.88 \pm 3.58$ & $1.67 \pm 0.40$ & $1.74 \pm 0.60$ \\
\hline
\end{tabular}




\begin{tabular}{|c|c|c|c|c|}
\hline Yield Stress (MPa) * & $127.47 \pm 33.29$ & $160.61 \pm 26.97$ & $83.44 \pm 17.83$ & $79.32 \pm 18.20$ \\
\hline Ultimate Stress (MPa) * & $141.64 \pm 27.35$ & $166.00 \pm 28.60$ & $98.65 \pm 20.20$ & $98.74 \pm 23.88$ \\
\hline Strain to Yield $(\mu \varepsilon)^{*}$ & $13699 \pm 2730$ & $15814 \pm 2902$ & $10456 \pm 2494$ & $11214 \pm 1873$ \\
\hline Total Strain $(\mu \varepsilon)^{*}$ & $37290 \pm 11853$ & $36561 \pm 15556$ & $16269 \pm 3909$ & $17704 \pm 2953$ \\
\hline Modulus (GPa) * & $9.60 \pm 1.14$ & $11.01 \pm 1.81$ & $8.26 \pm 2.65$ & $7.60 \pm 2.27$ \\
\hline Resilience (MPa) * & $0.95 \pm 0.42$ & $1.33 \pm 0.43$ & $0.45 \pm 0.13$ & $0.46 \pm 0.13$ \\
\hline Toughness (MPa) * & $3.47 \pm 1.37$ & $3.55 \pm 1.92$ & $0.95 \pm 0.30$ & $1.05 \pm 0.34$ \\
\hline
\end{tabular}

Values are presented as mean \pm standard deviation. WT- wild-type; oim-osteogenesis imperfecta. In the property column, * indicates a main effect of disease and + indicates a main effect of treatment.

\section{Fracture Toughness Testing}

Oim mice had significantly lower stress intensity factors at yielding ( $\left.\mathrm{K}_{\text {init }}\right)$, maximum load ( $\left.\mathrm{K}_{\text {max load }}\right)$ and failure $\left(\mathrm{K}_{\text {inst }}\right)$ compared to WT $(\mathrm{p}<0.0001)$. Treatment with RAL led to a significantly higher $\mathrm{K}_{\max \text { load }}(\mathrm{p}=0.0458)$ in both genotypes $(+8.43 \%$ in WT and $+4.19 \%$ in oim; Table 5).

Table 5: Fracture toughness of the femoral midshaft (MPa $\sqrt{\mathrm{mm}})$

\begin{tabular}{|l|c|c|c|c|}
\hline \multirow{2}{*}{} & \multicolumn{2}{|c|}{ WT } & \multicolumn{2}{c|}{ OIM } \\
\cline { 2 - 5 } & Control $(n=8)$ & Raloxifene $(n=7)$ & Control $(n=9)$ & $2.10 \pm 0.42$ \\
\hline Initiation Toughness* & $3.85 \pm 0.27$ & $4.10 \pm 0.37$ & $2.04 \pm 0.20$ & $2.24 \pm 0.43$ \\
\hline Maximum Load Toughness * + & $4.51 \pm 0.33$ & $4.89 \pm 0.38$ & $2.15 \pm 0.19$ & $2.56 \pm 0.60$ \\
\hline Instability Toughness * & $5.22 \pm 0.91$ & $5.27 \pm 0.94$ & $2.89 \pm 0.59$ & \\
\hline
\end{tabular}

Values are presented as mean \pm standard deviation. WT- wild-type; oim-osteogenesis imperfecta. In the property column, * indicates a main effect of disease and + indicates a main effect of treatment.

\section{DISCUSSION}

Osteogenesis imperfecta $(\mathrm{OI})$ is a debilitating musculoskeletal condition that presents unique treatment challenges due to the combination of bone mass, geometry, and material 
property deficits. The predominant pharmacological approach for addressing skeletal fragility has been to enhance bone mass/geometry and although successful in some regards, the preclinical literature clearly shows that adding bone mass is insufficient to completely normalize bone mechanical properties $[12,13,21-23,25,31,32]$. In the current study we demonstrated that raloxifene, which is known to improve bone mass/geometry and quality, positively affects oim bone through both cellular and non-cellular mechanisms. This suggests a novel approaches for offsetting the skeletal fragility of OI.

Ex vivo mechanical testing of 12 week old female mouse femurs in experiment 1 confirmed what numerous other papers have documented - the presence of significant mechanical deficits in oim mice compared to wild-type animals [8,10,21,25,33-35]. To test the hypothesis that raloxifene could rescue this mechanical phenotype, contralateral tibia were soaked in raloxifene, based on previous studies showing this was sufficient to increase skeletal hydration and mechanical properties through non-cellular mechanisms [28]. Soaking produced minimal effects on ultimate load or stiffness, but significant main effects on total displacement and energy to fracture (Figure 1 and Table 1). This is consistent with our previous work showing that raloxifene exposure mainly affects post-yield properties $[28,36]$. These data provide evidence that oim bone, like normal bone, can be positively affected by raloxifene through a non-cellular mechanism as the previously frozen tissue is void of viable cells. Based on our previous work we hypothesize that these beneficial effects within the matrix are the result of alterations in hydration. Hydration is known to play a key role in mechanical properties [37-39] and is modulated both in vitro and in vivo by raloxifene $[28,29]$. Unfortunately, hydration was not assessed in these bones due to technical challenges of working with such small amounts of tissue in the mouse bone. Future work should aim to assess hydration properties with and without interventions in pre-clinical models and hydration in patients through non-invasive methods [40,41].

The encouraging results from the soaking experiment translated to the in vivo experiment 
in which wild type and oim animals were treated with raloxifene for eight weeks. Much to our surprise, nearly half of the vehicle-treated oim animals had fractured femora at the time of sacrifice (16 weeks of age). In contrast, only $20 \%$ of the femurs in raloxifene-treated animals were fractured - representing a statistically significant, and in our opinion clinically meaningful, $58 \%$ reduction in the incidence of fracture. Spontaneous fractures are described in the homozygous oim mouse $[10,42]$, and have been characterized in several studies $[42,43,44]$. Although we did not count fractures at 8 weeks when treatment began, other studies which did characterize femoral fractures at 8 weeks of age suggest that between $22 \%$ and $27 \%$ of bones were fractured $[43,44]$. These numbers are close to the numbers seen in our RAL-treated mice at 16 weeks ( 6 of 30 bones, $20 \%$ ). Assuming that the oim mice allocated to each treatment group had comparable numbers of fractures at 8 weeks, this suggests that RAL may have prevented most additional fractures which would have occurred during the treatment period. Given that spontaneous fracture is exactly the phenotype we are most interested in treating clinically, our finding of significant reductions with a pharmacological treatment (raloxifene) represents a novel and exciting result.

Our imaging-based assessment of skeletal properties confirm work of others by showing oim animals have lower DXA-based BMD and CT-based trabecular bone volume and cortical bone area. Eight weeks of raloxifene treatment resulted in higher total body and femoral BMD, and higher trabecular bone volume in both genotypes (Figure 3). There were no significant interactions for any imaging measure, indicating that raloxifene was similarly effective in both wildtype and oim animals. Although raloxifene resulted in significantly higher bone mass compared to vehicle-treated oim animals, the values remained below those of untreated wild-type animals. This is in contrast to work with both bisphosphonates and anti-sclerostin antibody which have been shown to normalize bone mass back to wild-type levels in the oim $[22,23,42,45]$ and Brtl/+ models of OI [21,25]. Bisphosphonates are a more potent anti-resorptive agent compared to raloxifene, and anti-sclerostin antibody is a potent anabolic agent - thus the ability of these agents to enhance bone mass to a greater degree than raloxifene is not surprising. The potential to 
combine raloxifene with a more potent enhancer of bone mass represents an exciting next step.

The mechanical phenotype of oim animals, based on both four-point bending of the tibia and fracture mechanics testing of the femur, were consistent with previous work - showing reduced values for almost all parameters [8,21-23,25,33,34]. Overall, the effect of in vivo raloxifene treatment on the ex-vivo mechanical phenotype was unimpressive (Tables 4 and 5). While there was a significant main effect of raloxifene on $\mathrm{K}_{\max }$ load, indicative of a bone that necessitates a higher load to propagate an existing crack, this appears mainly driven by wild-type animals. The results of our four point bending tests contrast with the fact that there was a significant reduction in fracture. Although we do not have a clear explanation for these contrasting results, we present several hypotheses that could be tested in future studies. First, it's possible that the lower number of fractures in raloxifene-treated animals is a spurious result, yet given the effects seen in experiment 1 , and the previous work with raloxifene, this seems unlikely. An alternative explanation is that the effects observed in the two experiments are unrelated - the in vitro experiment changes being due to altered hydration and the in vivo experiment results being driven by some other change (altered activity level of mice, small changes in cortical thickness as two examples). This is a plausible explanation as the mechanisms could differ between males and females, or the local concentration of drug exposure could be different.

Raloxifene is an FDA approved therapy for treatment of post-menopausal osteoporosis. It's efficacy in fracture risk reduction in this patient population is clear, and appears to be due to a combination of traditional mechanisms (reduction of bone resorption) [26-28] and non-traditional mechanism (altered skeletal hydration) [26-28]. Despite this, there are also notable side effects (thrombosis and estrogenic features) that could pose challenges for translation into patients with OI (particularly children) [46]. Yet the results of the current study show, for the first time, the conceptual framework for a novel approach to treating $\mathrm{OI}$ - targeting the collagen matrix with a pharmacological agent. Ongoing work in our lab is aimed at developing novel compounds that impart benefits to matrix properties without having any estrogen receptor interaction. We 
hypothesize that such a compound, perhaps combined with an agent that suppresses osteoclast activity such as bisphosphonates, could represent a viable treatment regimen that targets both bone quantity and quality in OI.

There are some limitations of our study. We treated animals that were still actively growing, thus all of our effects are due to interactions between drug treatment and growth. We chose this timeline to match other $\mathrm{Ol}$ interventional treatments and to mimic an intervention in children with OI. Fracture incidence was not assessed at 8 weeks when the study began and, as such, it is possible that the oim treatment groups were unintentionally unbalanced at the start of the study. However, the number of fractures in untreated oim mice at 16 weeks of age here (13 or 27 or $48 \%$ ) is comparable to the number seen in untreated mice in a previous study at 12 weeks of age (53\%, [43]). Regardless, future studies will include a baseline measure of fracture prior to treatment. We studied only one dose of raloxifene, and only one sex for the in vivo treatment study. These are clearly variables that could be modified in future work and it is possible that the disparate results in mechanics between experiments 1 and 2 are due to the different sexes or drug exposures used for those studies. We chose our in vivo raloxifene dose based on matching the $\mathrm{mg} / \mathrm{kg}$ dose used in humans and the in vitro dose based on our previous work showing efficacy - yet we do not know that these provide similar drug exposure to the bone. While our sample size was sufficient to detect significant effects in many parameters, it may be necessary to have larger groups in order to detect differences in traditional mechanical properties. Finally, more detailed skeletal analyses, such as histological measures and those of bone quality (hydration/collagen/mineralization) would have been useful, yet these were beyond the scope of this early proof-of-concept study.

In conclusion, the current study shows beneficial effects of raloxifene exposure to bone properties in the setting of $\mathrm{Ol}$. Although the direct mechanism is unclear, changes translate to a significant reduction in the number of long bone fractures in this animal model of osteogenesis imperfecta. 


\section{EXPERIMENTAL PROCEDURES}

All animal procedures were performed with prior approval from the Indiana University Purdue University Science Animal Research Center (SARC) and Indiana University School of Medicine Institutional Animal Care and Use Committee (IACUC).

\section{Experiment Design \#1: Ex vivo Effects of Raloxifene}

Freshly frozen tibiae from a previous study [8] were used to assess non-cellular effects of raloxifene. Paired tibiae from 12 week old homozygous oim (B6C3Fe a/a-Col1a2oim/Col1a2oim; $\mathrm{n}=9)$ and $\mathrm{WT}(\mathrm{B} 6 \mathrm{C} 3 \mathrm{FeF} 1 / \mathrm{J}$ a/a; $\mathrm{n}=15)$ female mice [10] were incubated at $37^{\circ} \mathrm{C}$ in PBS or $2 \mu \mathrm{M}$ raloxifene supplemented with $1 \%$ Pen/Strep for 13 days (solution changed every $2-3$ day) as previously described [28]. After soaking, the bones were mechanically tested to failure in fourpoint bending ( $9 \mathrm{~mm}$ bottom support and $3 \mathrm{~mm}$ loading span) at a displacement rate of 0.025 $\mathrm{mm} / \mathrm{sec}$ following standard protocols [47]. Bending occurred in the medial-lateral direction with the medial side in tension. Samples remained hydrated with PBS while testing.

\section{Experiment Design \#2: In vivo Effects of Raloxifene}

Seven week old homozygous oim (B6C3Fe a/a-Col1a2oim/Col1a2oim) and WT (B6C3FeF1/J a/a) male mice were obtained from Jackson Laboratory. After one week of acclimation, mice were injected 5 days per week for eight weeks with saline vehicle (VEH; $n=8$ WT and $n=14$ oim) or $0.5 \mathrm{mg} / \mathrm{kg}$ raloxifene (RAL; $\mathrm{n}=8 \mathrm{WT}$ and $\mathrm{n}=15$ oim). This dose was chosen to approximate the clinical dose on a $\mathrm{mg} / \mathrm{kg}$ basis and have been used previously in pre-clinical studies $[29,36]$. At 16 weeks of age, the mice were euthanized by $\mathrm{CO}_{2}$ inhalation. Dual-energy $\mathrm{X}-$ 
ray absorptiometry (DXA) was performed with the whole body, femur, and L4-L5 vertebrae as regions of interest $(\mathrm{ROI})$. Following $\mathrm{DXA}$, the femurs and tibiae were removed, wrapped in salinesoaked gauze, and stored at $-20^{\circ} \mathrm{C}$ until analysis.

Microcomputed Tomography (uCT) Analysis of Femurs

Both femurs from each mouse were scanned while hydrated at a $9.8 \mu \mathrm{m}$ voxel size using a Bruker 1172 uCT system (176 mA, 0.5 mm Aluminum filter). Calibration was performed daily using two hydroxyapatite phantoms to convert grayscale values into $\mathrm{g} / \mathrm{cm}^{3}$. Images were then reconstructed for cortical and trabecular analyses. The projection scans of both left and right femora were used to assess the incidence of fracture. Three of the four authors examined the projection views and scored them as intact or fractured. When projection images were ambiguous of fracture, the reconstructed images were used for determination. Bones with fractures were excluded from further CT and mechanical analysis. For cortical analysis, a standard ROI was analyzed at $40 \%$ of the bone's length measured from the distal end. At this location, seven transverse slices were analyzed (approximately $69 \mu \mathrm{m}$ in length). Tissue mineral density (TMD) was calculated using vendor-supplied software (CTan). A standard binary threshold was then applied to the slices and geometric properties were calculated using a custom MATLAB code, as previously described [48]. Trabecular analysis was performed in the distal metaphysis on an ROI defined as $10 \%$ of the total bone length, beginning at the proximal end of the distal growth plate and extending proximally. Within that region, cancellous bone was segmented from the cortical shell using a custom MATLAB script. Geometric parameters were determined using vendorsupplied software (CTan).

Fracture Toughness Testing of Femurs 
Following $\mathrm{uCT}$, fracture toughness of the femurs was assessed following a linear elastic fracture mechanics approach $[33,49]$. Due to the incidence of fracture in the oim groups, a combination of left and right femurs was used. However, only one bone was tested per mouse. A notch was made on the anterior surface of the femur using a scalpel blade pasted with a $100 \mu \mathrm{m}$ diamond suspension. The notch entered the medullary cavity, but did not extend past the midpoint of the bone. Notched femurs were then tested to failure in 3 point bending at $0.001 \mathrm{~mm} / \mathrm{sec}$ with the loading point located directly above the notch. Following testing, bones were cleaned of marrow and dehydrated through graded ethanol (70-100\%) for scanning electron microscopy (SEM). SEM images of the fracture surface were used to obtain crack angles which, in conjunction with geometric properties from the UCT data, allowed for the calculation of fracture instability at $5 \%$ secant $\left(K_{\text {init }}\right)$, maximum load ( $\left.K_{\max \text { load }}\right)$, and failure load $\left(K_{\text {inst }}\right)$.

\section{Whole Bone Mechanical Testing of Tibiae}

Prior to whole bone mechanical testing, the left tibia from each mouse was scanned by UCT (16.8 $\mu \mathrm{m}$ voxel size) using methods described above. A lower resolution was used for the tibia as only cortical properties were of interest to allow for normalization of force-displacement data. The bones were then tested to failure in four-point bending as described in experiment 1. Following fracture, the location of the site of fracture was recorded and the $\mu \mathrm{CT}$ data were used to obtain geometric properties at the fracture location. A $5 \%$ loss in secant stiffness was used to define the yield point. Force-displacement data were normalized to stress-strain using standard beam bending equations and the data were analyzed using a custom MATLAB script, as previously described [48].

\section{Statistical Analysis}


All data were checked for assumptions of normality and homogeneity of variance, and violations were corrected using transformations. For in vitro soaking experiments, statistics consisted of a repeated measures ANOVA to observe within-subject (main effect of treatment) and between-subject (main effect of disease) results $(p<0.05)$. In the case of interactions, pairwise t-tests (paired for effect of treatment; unpaired for effect of disease) were performed and a Bonferroni correction was applied ( $p<0.0125$ was considered significant). For the in vivo treatment experiments, a two-Way ANOVA was performed to assess the main effects of disease and treatment ( $p<0.05$ was considered significant). Because of the staggered arrival of animals in our facility, the date of arrival was blocked as a nuisance factor. For the fracture assessment data, a Fisher's Exact Test was used to compare the proportion of fractures in oim VEH versus RAL as none of the WT mice exhibited fractures.

\section{Acknowledgements}

This work was supported by the IUPUI-BBRC funding mechanism and S10-RR023710 (microCT equipment grant). We thank Drew Brown for his invaluable role in animal dosing, tissue dissections, CT scanning and analysis. 


\section{References}

[1] D.J. McBride, J.R. Shapiro, Confirmation of a G nucleotide deletion in the Cola-2 gene of mice with the osteogenesis imperfecta mutation, Genomics. 20 (1994) 135-137. doi:10.1006/geno.1994.1141.

[2] J.C. Marini, A. Forlino, W.A. Cabral, A.M. Barnes, J.D. San Antonio, S. Milgrom, et al., Consortium for osteogenesis imperfecta mutations in the helical domain of type I collagen: regions rich in lethal mutations align with collagen binding sites for integrins and proteoglycans, Hum. Mutat. 28 (2007) 209-221. doi:10.1002/humu.20429.

[3] A.D. Kemp, C.C. Harding, W.A. Cabral, J.C. Marini, J.M. Wallace, Effects of tissue hydration on nanoscale structural morphology and mechanics of individual Type I collagen fibrils in the Brtl mouse model of Osteogenesis Imperfecta, Journal of Structural Biology. 180 (2012) 428-438. doi:10.1016/j.jsb.2012.09.012.

[4] J.M. Wallace, B.G. Orr, J.C. Marini, M.M.B. Holl, Journal of Structural Biology, Journal of Structural Biology. 173 (2011) 146-152. doi:10.1016/j.jsb.2010.08.003.

[5] U. Vetter, M.A. Weis, M. Mörike, E.D. Eanes, D.R. Eyre, Collagen crosslinks and mineral crystallinity in bone of patients with osteogenesis imperfecta, J Bone Miner Res. 8 (1993) 133-137. doi:10.1002/jbmr.5650080203.

[6] H. Stöss, P. Freisinger, Collagen fibrils of osteoid in osteogenesis imperfecta: morphometrical analysis of the fibril diameter, Am. J. Med. Genet. 45 (1993) 257-257. doi:10.1002/ajmg.1320450220.

[7] N.P. Camacho, L. Hou, T.R. Toledano, W.A. Ilg, C.F. Brayton, C.L. Raggio, et al., The material basis for reduced mechanical properties in oim mice bones, J Bone Miner Res. 14 (1999) 264-272. doi:10.1359/jbmr.1999.14.2.264.

[8] Z.R. Bart, M.A. Hammond, J.M. Wallace, Multi-scale analysis of bone chemistry, morphology and mechanics in the oim model of osteogenesis imperfecta, Connective Tissue Research. 55 (2015) 4-8. doi:10.3109/03008207.2014.923860.

[9] Sathyanarayanan, ANIMAL MODELS OF OSTEOGENESIS IMPERFECTA, (2013) 114.

[10] S.D. Chipman, H.O. Sweet, D.J. McBride, M.T. Davisson, S.C. Marks, A.R. Shuldiner, et al., Defective pro alpha 2(I) collagen synthesis in a recessive mutation in mice: a model of human osteogenesis imperfecta, Proceedings of the National Academy of Sciences of the United States of America. 90 (1993) 1701-1705.

[11] D.O. Sillence, A. Senn, D.M. Danks, Genetic heterogeneity in osteogenesis imperfecta, J. Med. Genet. 16 (1979) 101-116. doi:10.1136/jmg.16.2.101.

[12] F.H. Glorieux, Experience With Bisphosphonates in Osteogenesis Imperfecta, Pediatrics. 119 (2007) S163-S165. doi:10.1542/peds.2006-2023I.

[13] F.H. Glorieux, N.J. Bishop, H. Plotkin, G. Chabot, G. Lanoue, R. Travers, Cyclic administration of pamidronate in children with severe osteogenesis imperfecta, N. Engl. J. Med. 339 (1998) 947-952. doi:10.1056/NEJM199810013391402.

[14] F. Rauch, C.F. Munns, C. Land, M. Cheung, F.H. Glorieux, Risedronate in the Treatment of Mild Pediatric Osteogenesis Imperfecta: A Randomized PlaceboControlled Study, J Bone Miner Res. 24 (2009) 1282-1289. doi:10.1359/jbmr.090213.

[15] N. Bishop, R. Harrison, F. Ahmed, N. Shaw, R. Eastell, M. Campbell, et al., A randomized, controlled dose-ranging study of risedronate in children with moderate and severe osteogenesis imperfecta, J Bone Miner Res. 25 (2009) 32-40.

doi:10.1359/jbmr.090712.

[16] D. Gatti, F. Antoniazzi, R. Prizzi, V. Braga, M. Rossini, L. Tatò, et al., Intravenous Neridronate in Children With Osteogenesis Imperfecta: A Randomized Controlled Study, J Bone Miner Res. 20 (2004) 758-763. doi:10.1359/JBMR.041232.

[17] R. Sakkers, D. Kok, R. Engelbert, A. van Dongen, M. Jansen, H. Pruijs, et al., Skeletal 
effects and functional outcome with olpadronate in children with osteogenesis imperfecta: a 2-year randomised placebo-controlled study, The Lancet. 363 (2004) 1427-1431. doi:10.1016/S0140-6736(04)16101-1.

[18] A.D. Letocha, H.L. Cintas, J.F. Troendle, J.C. Reynolds, C.E. Cann, E.J. Chernoff, et al., Controlled Trial of Pamidronate in Children With Types III and IV Osteogenesis Imperfecta Confirms Vertebral Gains but Not Short-Term Functional Improvement, J Bone Miner Res. 20 (2005) 977-986. doi:10.1359/JBMR.050109.

[19] L.M. Ward, F. Rauch, M.P. Whyte, J. D'Astous, P.E. Gates, D. Grogan, et al., Alendronate for the Treatment of Pediatric Osteogenesis Imperfecta: A Randomized Placebo-Controlled Study, Journal of Clinical Endocrinology \& Metabolism. 96 (2011) 355-364. doi:10.1210/jc.2010-0636.

[20] P.C.S.R.B.D. Dwan K, Bisphosphonate therapy for osteogenesis imperfecta (Review), (2014) 1-74.

[21] T.E. Uveges, K.M. Kozloff, J.M. Ty, F. Ledgard, C.L. Raggio, G. Gronowicz, et al., Alendronate Treatment of the Brtl Osteogenesis Imperfecta Mouse Improves Femoral Geometry and Load Response Before Fracture but Decreases Predicted Material Properties and Has Detrimental Effects on Osteoblasts and Bone Formation, J Bone Miner Res. 24 (2009) 849-859. doi:10.1359/jbmr.081238.

[22] B.M. Misof, P. Roschger, T. Baldini, C.L. Raggio, V. Zraick, L. Root, et al., Differential effects of alendronate treatment on bone from growing osteogenesis imperfecta and wild-type mouse, Bone. 36 (2005) 150-158. doi:10.1016/j.bone.2004.10.006.

[23] R. Bargman, R. Posham, A.L. Boskey, E. DiCarlo, C. Raggio, N. Pleshko, Comparable outcomes in fracture reduction and bone properties with RANKL inhibition and alendronate treatment in a mouse model of osteogenesis imperfecta, Osteoporos Int. 23 (2011) 1141-1150. doi:10.1007/s00198-011-1742-7.

[24] M.R. Allen, D.B. Burr, Bisphosphonate effects on bone turnover, microdamage, and mechanical properties: what we think we know and what we know that we don't know, Bone. 49 (2011) 56-65. doi:10.1016/j.bone.2010.10.159.

[25] B.P. Sinder, M.M. Eddy, M.S. Ominsky, M.S. Caird, J.C. Marini, K.M. Kozloff, Sclerostin antibody improves skeletal parameters in a Brtl/+ mouse model of osteogenesis imperfecta, J Bone Miner Res. 28 (2012) 73-80. doi:10.1002/jbmr.1717.

[26] R.R. Recker, B.H. Mitlak, X. Ni, J.H. Krege, Long-term raloxifene for postmenopausal osteoporosis, Curr Med Res Opin. 27 (2011) 1755-1761.

doi:10.1185/03007995.2011.606312.

[27] E. Seeman, G.G. Crans, A. Diez-Perez, K.V. Pinette, P.D. Delmas, Anti-vertebral fracture efficacy of raloxifene: a meta-analysis, Osteoporos Int. 17 (2005) 313-316. doi:10.1007/s00198-005-2030-1.

[28] M.A. Gallant, D.M. Brown, M. Hammond, J.M. Wallace, J. Du, A.C. Deymier-Black, et al., Bone cell-independent benefits of raloxifene on the skeleton: A novel mechanism for improving bone material properties, Bone. 61 (2014) 191-200.

doi:10.1016/j.bone.2014.01.009.

[29] M.R. Allen, P.R. Territo, C. Lin, S. Persohn, L. Jiang, A.A. Riley, et al., In Vivo UTE-MRI Reveals Positive Effects of Raloxifene on Skeletal-Bound Water in Skeletally Mature Beagle Dogs, J Bone Miner Res. 30 (2015) 1441-1444. doi:10.1002/jbmr.2470.

[30] C.L. Newman, A. Creecy, M. Granke, J.S. Nyman, N. Tian, M.A. Hammond, et al., Raloxifene improves skeletal properties in an animal model of chronic kidney disease, Kidney Int. (2015) In press.

[31] E.S. Orwoll, J. Shapiro, S. Veith, Y. Wang, J. Lapidus, C. Vanek, et al., Evaluation of teriparatide treatment in adults with osteogenesis imperfecta, J Clin Invest. (2014). doi:10.1172/JCI71101.

[32] H. Hoyer-Kuhn, O. Semler, E. Schoenau, Effect of denosumab on the growing skeleton 
in osteogenesis imperfecta, J. Clin. Endocrinol. Metab. 99 (2014) 3954-3955. doi:10.1210/jc.2014-3072.

[33] A. Carriero, E.A. Zimmermann, A. Paluszny, S.Y. Tang, H. Bale, B. Busse, et al., How tough is brittle bone? Investigating osteogenesis imperfecta in mouse bone, J Bone Miner Res. 29 (2014) 1392-1401. doi:10.1002/jbmr.2172.

[34] K.M. Kozloff, A. Carden, C. Bergwitz, A. Forlino, T.E. Uveges, M.D. Morris, et al., Brittle IV mouse model for osteogenesis imperfecta IV demonstrates postpubertal adaptations to improve whole bone strength, J Bone Miner Res. 19 (2004) 614-622.

doi:10.1359/JBMR.040111.

[35] B.P. Sinder, J.D. Salemi, M.S. Ominsky, M.S. Caird, J.C. Marini, K.M. Kozloff, Rapidly growing Brtl/+ mouse model of osteogenesis imperfecta improves bone mass and strength with sclerostin antibody treatment, Bone. 71 (2015) 115-123. doi:10.1016/j.bone.2014.10.012.

[36] M. Allen, H. Hogan, W. Hobbs, A. Koivuniemi, M. Koivuniemi, D. Burr, Raloxifene enhances material-level mechanical properties of femoral cortical and trabecular bone, Endocrinology. 148 (2007) 3908-3913.

[37] M. Granke, M.D. Does, J.S. Nyman, The Role of Water Compartments in the Material Properties of Cortical Bone, Calcif Tissue Int. 97 (2015) 292-307. doi:10.1007/s00223015-9977-5.

[38] E.Y. Chang, W.C. Bae, H. Shao, R. Biswas, S. Li, J. Chen, et al., Ultrashort echo time magnetization transfer (UTE-MT) imaging of cortical bone, NMR Biomed. 28 (2015) 873-880. doi:10.1002/nbm.3316.

[39] J.S. Nyman, Q. Ni, D.P. Nicolella, X. Wang, Measurements of mobile and bound water by nuclear magnetic resonance correlate with mechanical properties of bone, Bone. 42 (2008) 193-199. doi:10.1016/j.bone.2007.09.049.

[40] M.K. Manhard, R.A. Horch, D.F. Gochberg, J.S. Nyman, M.D. Does, In Vivo Quantitative MR Imaging of Bound and Pore Water in Cortical Bone, Radiology. (2015) 140336-9. doi:10.1148/radiol.2015140336.

[41] A. Techawiboonwong, H.K. Song, M.B. Leonard, F.W. Wehrli, Cortical Bone Water: In Vivo Quantification with Ultrashort Echo-Time MR Imaging, Radiology. 248 (2008) 824833. doi:10.1148/radiol.2482071995.

[42] N.P. Camacho, C.L. Raggio, S.B. Doty, L. Root, V. Zraick, W.A. Ilg, et al., A Controlled Study of the Effects of Alendronate in a Growing Mouse Model of Osteogenesis Imperfecta, Calcif Tissue Int. 69 (2001) 94-101. doi:10.1007/s002230010045.

[43] M. Vanleene, Z. Saldanha, K.L. Cloyd, G. Jell, G. Bou-Gharios, J.H.D. Bassett, et al., Transplantation of human fetal blood stem cells in the osteogenesis imperfecta mouse leads to improvement in multiscale tissue properties, Blood. 117 (2011) 1053-1060. doi:10.1182/blood-2010-05-287565.

[44] P.V. Guillot, O. Abass, J.H.D. Bassett, S.J. Shefelbine, G. Bou-Gharios, J. Chan, et al., Intrauterine transplantation of human fetal mesenchymal stem cells from first-trimester blood repairs bone and reduces fractures in osteogenesis imperfecta mice, Blood. 111 (2008) 1717-1725. doi:10.1182/blood-2007-08-105809.

[45] K.D. Evans, S.T. Lau, A.M. Oberbauer, R.B. Martin, Alendronate affects long bone length and growth plate morphology in the oim mouse model for Osteogenesis Imperfecta, Bone. 32 (2003) 268-274. doi:10.1016/S8756-3282(02)00974-2.

[46] I.R. Reid, Efficacy, effectiveness and side effects of medications used to prevent fractures, J Intern Med. 277 (2015) 690-706. doi:10.1111/joim.12339.

[47] J. Wallace, R. Rajachar, X. Chen, S. Shi, M. Allen, S. Bloomfield, et al., The mechanical phenotype of biglycan-deficient mice is bone-and gender-specific, Bone. 39 (2006) $106-116$.

[48] A.G. Berman, C.A. Clauser, C. Wunderlin, M.A. Hammond, J.M. Wallace, Structural and 
Mechanical Improvements to Bone Are Strain Dependent with Axial Compression of the Tibia in Female C57BL/6 Mice, PLoS ONE. 10 (2015) e0130504-16.

doi:10.1371/journal.pone.0130504.

[49] E.M. McNerny, B. Gong, M.D. Morris, D.H. Kohn, Bone Fracture Toughness and Strength Correlate With Collagen Cross-Link Maturity in a Dose-Controlled Lathyrism Mouse Model, J Bone Miner Res. 30 (2015) 455-464. doi:10.1002/jbmr.2356. 